CLINICAL STUDY

\title{
Raised serum TSH levels in patients with morbid obesity: is it enough to diagnose subclinical hypothyroidism?
}

\author{
Mario Rotondi, Paola Leporati, Antonella La Manna, Barbara Pirali, Teresa Mondello, Rodolfo Fonte, Flavia Magri \\ and Luca Chiovato \\ Unit of Internal Medicine and Endocrinology, Fondazione Salvatore Maugeri I.R.C.C.S., ISPESL Laboratory for Endocrine Disruptors, Chair of \\ Endocrinology, University of Pavia, Via S. Maugeri 10, I-27100 Pavia, Italy
}

(Correspondence should be addressed to L Chiovato; Email: lchiovato@fsm.it)

\begin{abstract}
Objective: Morbid obesity (body mass index (BMI) $\geq 40 \mathrm{~kg} / \mathrm{m}^{2}$ ) is associated with thyroid function disturbances, with a high rate of subclinical hypothyroidism $(\mathrm{SH})$ being the most consistently reported. We evaluated the circulating thyroid function parameters in morbid obese patients and related the results to the presence of circulating thyroid antibodies (Thyr-Ab).

Design and methods: Morbid obese patients were consecutively enrolled $(n=350)$. Two control groups were used: control group (CG)1, healthy normo-weight subjects $(n=50)$; CG2, normo-weight patients with SH $(n=56)$ matched for TSH with the obese patients with SH. Serum levels of free triiodothyronine $\left(\mathrm{FT}_{3}\right)$, free thyroxine $\left(\mathrm{FT}_{4}\right)$, TSH, antithyroglobulin antibodies, and antithyroperoxidase antibodies were measured in all patients.

Results: i) Compared with CG1, obese patients having thyroid function parameters in the normal range and negative Thyr-Ab showed significantly higher serum TSH and lower free thyroid hormones levels, but a similar $\mathrm{FT}_{4} / \mathrm{FT}_{3}$ ratio; ii) $\mathrm{SH}$ was recorded in $13.7 \%$ obese patients; iii) compared with CG2, obese patients with untreated SH had a significantly lower rate of positive Thyr-Ab (32.1 vs $66.1 \%$; $P<0.005)$; iv) no gender prevalence was observed in SH obese patients with negative Thyr-Ab; and v) the comparison of the untreated SH patients (obese and normo-weight) with CG1 demonstrated that in $\mathrm{SH}$ obese subjects, unlike normo-weight $\mathrm{SH}$ patients, the $\mathrm{FT}_{3}$ levels were significantly lower. This resulted in a normal $\mathrm{FT}_{4} / \mathrm{FT}_{3}$ ratio in $\mathrm{SH}$ obese patients.

Conclusion: Thyroid autoimmunity is not a major cause sustaining the high rate of SH in morbid obese patients. In these patients, the diagnosis of SH itself, as assessed by a raised TSH alone, appears questionable.
\end{abstract}

European Journal of Endocrinology $160403-408$

\section{Introduction}

Obesity has become an epidemic condition in developed countries. In the USA, the prevalence of obesity (defined as a body mass index (BMI) $\geq 30 \mathrm{~kg} / \mathrm{m}^{2}$ ) has increased from $15.3 \%$ in 1995 to $23.9 \%$ in 2005 (1). Morbid obesity (BMI $\geq 40 \mathrm{~kg} / \mathrm{m}^{2}$ ) accounts for nearly $5.0 \%$ of all obese patients (2). The pathophysiology of obesity is complex and still poorly understood, but it includes genetic, environmental, behavioral, and psychological factors (3). Family studies suggest that heredity may explain up to $67 \%$ of the population variance in BMI (4). Obesity is associated with an increased risk of hypertension, diabetes, hyperlipidemia, sleep apnea, coronary heart disease, and stroke $(3,5)$. As a consequence, in developed countries, increasing rates of obesity may lead to a decline in the overall life expectancy $(6,7)$. Furthermore, the obesity-associated morbidity is economically damaging for society. Obesity, with its array of comorbidities, necessitates careful clinical assessment to identify underlying factors and to allow coherent management, which is of critical importance for the affected individuals (8).

In the past 10 years, several clinical studies evaluated the issue of hormonal changes associated with obesity $(9$, 10). Thyroid dysfunctions have been extensively investigated in obese subjects (11-16). Although previous studies consistently reported changes in thyroid function parameters in obese subjects, thus supporting the hypothesis of a regulation loop involving the pituitary, the thyroid, and the adipose tissue, $(17,18)$ quantitative and qualitative estimations of such changes lead to variable results in the different studies. These discrepant findings might be ascribed, at least in part, to the fact that most previous studies included patients with a wide range of overweight. Failure to select a specific degree of obesity would contrast with clinical and genetic evidences supporting the concept that obesity, throughout its gradient of severity, does not represent a continuous entity. As a consequence, obese patients 
with a BMI lower or greater than $40 \mathrm{~kg} / \mathrm{m}^{2}$ might show relevant differences as to specific clinical and physiopathological aspects. Thyroid function changes have not been extensively investigated in patients with morbid obesity. The only study specifically including patients with extreme obesity reported, when compared with normo-weight subjects, significant differences in the serum levels of $\mathrm{TSH}_{\mathrm{H}}$ and $\mathrm{FT}_{3}$, accompanied by a high rate of hypothyroidism (12). In the general adult population, the majority of cases of subclinical and overt hypothyroidism result from chronic autoimmune thyroiditis, the hallmark of which are circulating thyroid antibodies (Thyr-Ab). The role of thyroid autoimmunity as a cause of thyroid function abnormalities has not been extensively investigated in patients with morbid obesity. The aim of this study was to evaluate the serum concentrations of free thyroxine $\left(\mathrm{FT}_{4}\right)$, free triiodothyronine $\left(\mathrm{FT}_{3}\right)$, and TSH in a large cohort of patients with morbid obesity, and to relate their changes to the presence of circulating Thyr-Ab.

\section{Patients and methods}

\section{Subjects}

The study group encompassed patients with morbid obesity (BMI $\geq 40 \mathrm{~kg} / \mathrm{m}^{2}$ ) consecutively recruited in the Unit of Internal Medicine and Endocrinology of the Fondazione Maugeri. In detail, 350 patients (94 males and 256 females) were investigated by measuring their serum concentrations of $\mathrm{FT}_{4}, \mathrm{FT}_{3}$, TSH, antithyroglobulin $\mathrm{Ab}(\mathrm{Tg}-\mathrm{Ab})$, and antithyroperoxidase $\mathrm{Ab}$ (TPO$\mathrm{Ab})$. Their mean age $( \pm$ s.D. $)$ was $46.2 \pm 12.2$ years. Weight was measured to the nearest kilogram. Height was determined to the nearest centimeter. BMI was calculated as the weight $(\mathrm{kg})$ divided by the square of height $(\mathrm{m})$. None of the patients was treated with a hypocaloric diet.

\section{Controls}

Two control groups were used. The first control group (CG1) consisted of 50 sex- and age-matched healthy normo-weight subjects, in whom thyroid disorders had been excluded by a complete thyroid work-up. This included history; physical examination; measurement of serum $\mathrm{FT}_{4}, \mathrm{FT}_{3}$, TSH, Tg-Ab, and $\mathrm{TPO}-\mathrm{Ab}$; and thyroid ultrasonography.

A second CG2 included sex- and age-matched normoweight patients with overt and subclinical hypothyroidism (SH) seen in our outpatient clinic during the same period of time when the obese patients were recruited. In detail, two hypothyroid patients, having the same serum level of TSH as any individual obese patient with a raised TSH, were enrolled in a case-control-designed protocol. When more than two hypothyroid patients had the same serum TSH level as an obese subject, the choice was randomly performed.

SH was biochemically defined as a serum level of TSH above the normal range $(0.4-4.0 \mathrm{mIU} / \mathrm{l})$ with $\mathrm{FT}_{4}$ and $\mathrm{FT}_{3}$ concentrations within the normal reference range (19). Overt hypothyroidism was diagnosed when serum TSH was above the normal range and serum $\mathrm{FT}_{4}$ levels were found lower than the normal range.

Blood samples were drawn between 0800 and $0900 \mathrm{~h}$, after an overnight fast.

All subjects gave their informed consent to participate in the study, which was performed in accordance with the guidelines of the declaration of Helsinki.

\section{Serum assays}

Serum concentrations of $\mathrm{FT}_{4}$ (normal range: 8.0$19.0 \mathrm{pg} / \mathrm{ml}$ ), $\mathrm{FT}_{3}$ (normal range: $1.8-4.2 \mathrm{pg} / \mathrm{ml}$ ), and TSH (third-generation TSH assay; normal range: 0.4$4.0 \mathrm{mIU} / \mathrm{l}$ ) were measured using immunochemoluminescent assays by an automated analyzer (Immulite 2000; Diagnostic Products Corporation Cirrus, Los Angeles, CA, USA) employing commercial kits (Diagnostic Products Corporation). Serum concentrations of $\mathrm{Tg}-\mathrm{Ab}$ (normal range: $<60 \mathrm{U} / \mathrm{ml}$ ) and TPO-Ab (normal range: $<60 \mathrm{U} / \mathrm{ml}$ ) were measured using immunochemoluminescent assays employing commercial kits (Brahms, Hennigsdorf, Germany).

\section{Statistical analysis}

Statistical analysis was performed using SPSS software (SPSS, Inc., Evanston, IL, USA). Between-group comparisons were performed by Student's $t$-test for unpaired data and by Mann-Whitney $U$-test according to a normal or a nonparametric distribution of the variable tested. Correlation between two variables was ascertained by Pearson and Spearman's correlation tests, as appropriate. Frequencies among groups were compared by $\chi^{2}$ test with Fisher's correction, when appropriate. $P<0.05$ was considered statistically significant.

\section{Results}

\section{Thyroid function status in patients with morbid obesity}

The clinical, hormonal, and antibody features of all patients with morbid obesity are reported in Table 1. On first observation, 20 out of $350(5.7 \%)$ morbid obese patients were treated with $\mathrm{L}-\mathrm{T}_{4}$ for a previous diagnosis of hypothyroidism. Out of 350, $28(8.0 \%)$ were first discovered as having a raised TSH ranging from 4.1 to 19.9 (median 4.9) $\mathrm{mU} / \mathrm{l}$. Their median serum-free thyroid hormone levels were $10.5(5.0-16.0) \mathrm{pg} / \mathrm{ml}$ and $3.0(1.5-4.4) \mathrm{pg} / \mathrm{ml}$ for $\mathrm{FT}_{4}$ and $\mathrm{FT}_{3}$ respectively. 
Table 1 Baseline characteristics of obese patients.

\begin{tabular}{|c|c|c|}
\hline Characteristics/findings & Value & (\%) \\
\hline Number of patients & 350 & \\
\hline Age range (years) & $18-78$ & \\
\hline Mean age \pm S.D. & $46.2 \pm 12.2$ & \\
\hline \multicolumn{3}{|l|}{$\operatorname{Sex}(n)$} \\
\hline $\mathrm{M}$ & 94 & $(26.9)$ \\
\hline $\mathrm{F}$ & 256 & $(73.1)$ \\
\hline Mean body weight $(\mathrm{kg}) \pm$ s.D. & $128.6 \pm 21.9$ & \\
\hline Mean BMI $\left(\mathrm{kg} / \mathrm{m}^{2}\right) \pm$ s.D. & $48.8 \pm 6.7$ & \\
\hline Untreated euthyroid & 299 & $(85.4)$ \\
\hline $\begin{array}{l}\text { Euthyroid on } \mathrm{L}^{-\mathrm{T}_{4}} \text { (for a previous } \\
\text { diagnosis of hypothyroidism) }\end{array}$ & 20 & $(5.7)^{\prime}$ \\
\hline Untreated with TSH > $4.0 \mathrm{mU} / \mathrm{l}$ & 28 & $(8.0)$ \\
\hline $\begin{array}{l}\text { Euthyroid on ATD } \text { A (for a previous } \\
\text { diagnosis of hyperthyroidism) }\end{array}$ & 3 & $(0.8)$ \\
\hline Tg Ab positive & 18 & $(5.1)$ \\
\hline TPO Ab positive & 35 & $(10.0)$ \\
\hline Tg Ab and/or TPO Ab positive & 40 & (11.4) \\
\hline
\end{tabular}

${ }^{a}$ Anti-thyroid drugs.

All these 28 untreated obese patients, with the exception of one case, showed normal serum levels of $\mathrm{FT}_{4}$; thus their thyroid dysfunction would have been classified as SH. Overall, 48 out of $350(13.7 \%)$ obese patients would have been classified as having an untreated or a successfully replaced hypothyroidism.

Hyperthyroidism was by far less frequent in our cohort of patients, with only 3 patients out of 350 $(0.8 \%)$ being treated with anti-thyroid drugs for a previous diagnosis of hyperthyroidism.

Positive tests for circulating thyroid $\mathrm{Ab}(\mathrm{Tg}-\mathrm{Ab}$ and/or TPO-Ab) were found in 40 out of $350(11.4 \%)$ obese patients. In particular, 35 patients $(10.0 \%)$ were positive for TPO-Ab, while 18 patients $(5.1 \%)$ showed positive results for circulating $\mathrm{Tg}$-Ab. According to gender, 3 males out of $94(3.2 \%)$ and 37 females out of $256(14.4 \%)$ had positive tests for circulating thyroid Ab $(P<0.01)$.

\section{Comparison of thyroid function parameters between euthyroid obese and normo-weight subjects}

Two-hundred and eighty obese patients, being untreated with thyroid medications, had thyroid function parameters within the normal range and negative tests for Thyr-Ab. In this subgroup of euthyroid obese patients, with no humoral sign of thyroid autoimmunity, there was no significant correlation between serum thyroid function parameters and either BMI or body weight. The serum concentrations of $\mathrm{FT}_{4}$, $\mathrm{FT}_{3}$, and TSH observed in this group of morbid obese patients were compared with the correspondent values found in CG1, i.e. euthyroid normo-weight subjects with no evidence of thyroid autoimmunity $(n=50)$. Patients with morbid obesity showed significantly lower serum levels of $\mathrm{FT}_{4}$ and $\mathrm{FT}_{3}$, and higher serum concentrations of TSH, when compared with healthy controls.
Interestingly, the $\mathrm{FT}_{4} / \mathrm{FT}_{3}$ ratio was similar in the two groups (Table 2).

\section{Comparison of thyroid function parameters between TSH-matched obese and normo-weight patients with SH}

The serum levels of $\mathrm{FT}_{4}$ and $\mathrm{FT}_{3}$ were compared between the untreated patients with a raised serum TSH, either obese $(n=28)$ or normo-weight $(n=56)$. As indicated in the Materials and methods section, two normo-weight hypothyroid patients with an equivalent serum TSH level were used as controls (CG2) for each obese subject. Euthyroid normo-weight subjects (CG1) were used as a further control group. Results of $\mathrm{FT}_{4}$, $\mathrm{FT}_{3}$, and $\mathrm{FT}_{4} / \mathrm{FT}_{3}$ ratio in the three groups are shown in Table 3. When compared with normo-weight euthyroid subjects, both obese and normo-weight hypothyroid patients had significantly lower serum $\mathrm{FT}_{4}$ concentrations. On the other hand, the serum levels of $\mathrm{FT}_{3}$ displayed a different behavior, being significantly lower in hypothyroid obese subjects, but not in hypothyroid normo-weight patients, when compared with euthyroid normo-weight controls. As a consequence, the $\mathrm{FT}_{4} / \mathrm{FT}_{3}$ ratio, while being significantly lower in hypothyroid normo-weight patients, did not differ in hypothyroid obese subjects compared with euthyroid normo-weight controls.

\section{Evidence for humoral thyroid autoimmunity in morbid obese patients and in controls}

The overall prevalence of thyroid Ab positivity was $11.4 \%$ in the whole cohort of patients with morbid obesity. To further investigate this issue, all the 48 obese patients being classified as hypothyroid (i.e. those being treated with $\mathrm{L}_{-} \mathrm{T}_{4}$ for a previous diagnosis of hypothyroidism and those with a raised TSH in the absence of specific treatment) were considered together. The remaining 299 obese patients with normal thyroid function parameters constituted the euthyroid group. Hyperthyroid obese patients were excluded from this analysis.

Table 2 Comparison of circulating thyroid function parameters between euthyroid obese patients with negative tests for thyroid $A b$ and healthy normo-weight controls (CG1).

\begin{tabular}{lccc}
\hline & $\begin{array}{c}\text { Euthyroid } \\
\text { Ab-negative } \\
\text { obese patients }\end{array}$ & $\begin{array}{c}\text { Euthyroid } \\
\text { normo-weight } \\
\text { control subjects } \\
\text { (CG1) }\end{array}$ & P value \\
\hline Number of & 280 & 50 & \\
$\quad$ cases & & & \\
$\mathrm{FT}_{4}(\mathrm{ng} / \mathrm{dl})$ & $12.7 \pm 2.23$ & $13.9 \pm 2.17$ & 0.001 \\
$\mathrm{FT}_{3}(\mathrm{pg} / \mathrm{ml})$ & $3.08 \pm 0.47$ & $3.41 \pm 0.54$ & 0.0001 \\
$\mathrm{TSH}^{(\mathrm{mUl} / \mathrm{l})}$ & $1.8 \pm 0.83$ & $1.2 \pm 0.46$ & $<0.0001$ \\
$\mathrm{FT}_{4} / \mathrm{FT}_{3}$ & $4.20 \pm 0.94$ & $4.22 \pm 1.08$ & $\mathrm{NS}$ \\
\hline
\end{tabular}


Table 3 Comparison of serum-free thyroid hormones levels between healthy normo-weight controls (CG1), hypothyroid normo-weight patients (CG2), and hypothyroid obese patients.

\begin{tabular}{|c|c|c|c|c|c|}
\hline & $\begin{array}{c}\text { Euthyroid } \\
\text { normo-weight } \\
\text { controls (CG1) }\end{array}$ & $\begin{array}{l}\text { Normo-weight } \\
\text { controls with SH } \\
\text { (CG2) }\end{array}$ & $\begin{array}{l}P \text { value } \\
\text { versus CG1 }\end{array}$ & $\begin{array}{c}\text { Morbid obese } \\
\text { patients with SH }\end{array}$ & $\begin{array}{l}P \text { value } \\
\text { versus CG1 }\end{array}$ \\
\hline No. of cases & 50 & 56 & & 28 & \\
\hline $\mathrm{FT}_{3}(\mathrm{ng} / \mathrm{dl})$ & $3.44 \pm 0.53$ & $3.37 \pm 0.90$ & NS & $3.06 \pm 0.60$ & $<0.005$ \\
\hline $\mathrm{FT}_{4}(\mathrm{pg} / \mathrm{ml})$ & $13.79 \pm 2.17$ & $11.20 \pm 2.18$ & $<0.0001$ & $11.41 \pm 2.11$ & $<0.0001$ \\
\hline $\mathrm{TSH}(\mathrm{mUI} / \mathrm{l})$ & $1.28 \pm 0.51$ & $5.93 \pm 2.97$ & $<0.0001$ & $5.95 \pm 3.07$ & $<0.0001$ \\
\hline $\mathrm{FT}_{4} / \mathrm{FT}_{3}$ & $4.14 \pm 1.09$ & $3.49 \pm 0.92$ & $<0.01$ & $3.68 \pm 0.79$ & NS \\
\hline
\end{tabular}

As expected, the positivity rate for thyroid $\mathrm{Ab}$ was significantly higher in hypothyroid when compared with euthyroid obese patients. In detail, 19 out of 299 (6.35\%) euthyroid obese patients were positive for either $\mathrm{Tg}-\mathrm{Ab}$ or TPO-Ab, as opposed to 18 out of 48 (37.5\%) hypothyroid obese patients $(P<0.0001)$.

The relatively low prevalence of circulating thyroid $\mathrm{Ab}(37.5 \%)$ in obese hypothyroid patients prompted a further analysis, which was restricted to those patients who were untreated. The rate of thyroid Ab positivity in this group of obese patients was compared with that observed in TSH-matched normo-weight hypothyroid patients (CG2). No significant differences were observed as to serum $\mathrm{FT}_{4}, \mathrm{FT}_{3}$, and $\mathrm{FT}_{4} / \mathrm{FT}_{3}$ ratio between the two groups. On the other hand, the rate of positivity for circulating thyroid $\mathrm{Ab}$ in the 56 hypothyroid normoweight patients was $66.1 \%$, when compared with a significantly $(P<0.005)$ lower figure $(32.1 \%)$ found in the 28 hypothyroid obese patients (Fig. 1).

In the whole cohort of obese patients considered in the present study, the rate of hypothyroidism was significantly higher $(P<0.05)$ in females $(16.5 \%)$ when compared with males $(6.5 \%)$; however, the male/female ratio was much lower (half) than that expected. This relatively low female gender prevalence required further investigation. When only hypothyroid obese patients who were positive for circulating thyroid

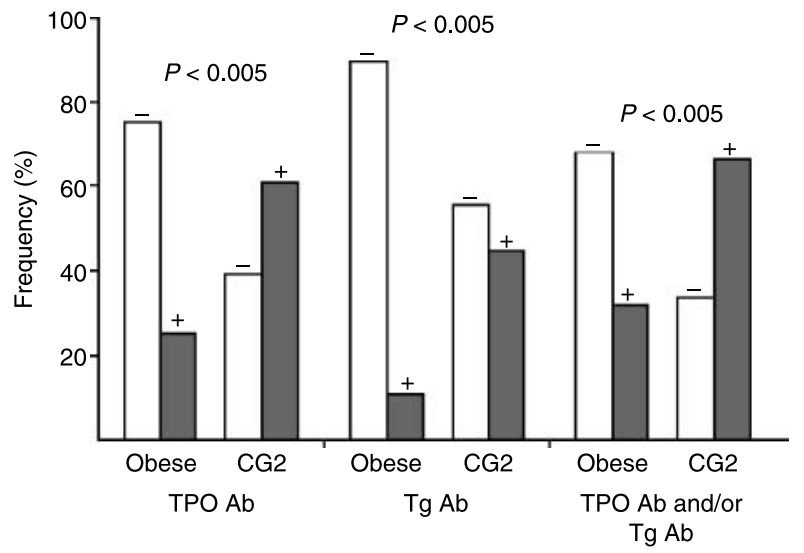

Figure 1 Positivity rate for thyroid antibodies in hypothyroid morbid obese patients and in hypothyroid TSH-matched normo-weight patients (CG2; +, positive test; -, negative test).
$\mathrm{Ab}$ were taken into account, a significant association was found between hypothyroidism and female sex. Indeed, the rate of hypothyroidism was $1.5 \%$ ( 1 case out of 87) among male patients versus $7.0 \%$ (16 cases out of 229 ) in females, with a male/female ratio of 1:4.7; $(P<0.05)$. On the other hand, when only hypothyroid patients with negative tests for thyroid $A b$ were taken into account, there was no significant difference in the gender prevalence, with 5 out of $91(5.5 \%)$ affected males and 26 out of 239 (10.9\%) affected females (NS).

\section{Discussion}

Thyroid function parameters and thyroid $\mathrm{Ab}$ findings were investigated in a large group of patients with morbid obesity (BMI $\geq 40 \mathrm{~kg} / \mathrm{m}^{2}$ ), and compared with euthyroid or hypothyroid control groups of normoweight subjects. The main results are hereby summarized and commented.

First of all, morbid obese patients showing serum levels of free thyroid hormones and TSH within the normal range and having negative tests for circulating $\mathrm{Tg}-\mathrm{Ab}$ and TPO-Ab displayed significantly lower serum levels of $\mathrm{FT}_{4}$ and $\mathrm{FT}_{3}$, and higher serum concentrations of TSH compared with normo-weight euthyroid subjects. However, the $\mathrm{FT}_{4} / \mathrm{FT}_{3}$ ratio did not differ between euthyroid obese patients and normo-weight subjects. Previous studies evaluating thyroid function parameters in obese patients reported discrepant findings. The most consistently reported observation was that, as opposed to healthy controls $(11,12,14-$ 16), the serum levels of TSH are higher in obese patients. Unlike $\mathrm{TSH}$, data regarding the circulating levels of free thyroid hormones are discrepant between different studies, which reported either increased or decreased serum concentrations of $\mathrm{FT}_{3}(11,12,14)$, with normal or decreased $\mathrm{FT}_{4} / \mathrm{FT}_{3}$ ratios $(12,14)$. Discrepancy is most likely due to the inclusion of patients with different degrees of obesity, as assessed by BMI, or different fasting plasma insulin concentrations, which were recently demonstrated to be positively related to $\mathrm{FT}_{3}$ levels, independently from plasma glucose concentration (20).

Indeed, the clinical and genetic peculiarities of patients with morbid obesity would suggest that the 
repercussions of body weight excess on thyroid function parameters should not be regarded as a continuous gradient, which implies that differences may exist between lower grades of overweight and morbid obesity.

The second relevant observation was that hypothyroid patients with morbid obesity showed an unexpectedly low rate of positive tests for thyroid $\mathrm{Ab}$ (21). This finding was also evident in untreated obese patients with a raised TSH, who, when compared with TSHmatched normo-weight hypothyroid patients, had a significantly lower rate of thyroid antibody positivity (32 vs $66 \%$ respectively). Thus, in the majority of morbid obese patients with a raised TSH, there was no evidence of humoral thyroid autoimmunity as a possible cause of their abnormal thyroid function. The finding of such a high rate of negative tests for thyroid $\mathrm{Ab}$ in obese patients with hypothyroidism is not limited to our study (12) and deserves a specific discussion.

The prevalence of positive tests for circulating thyroid $\mathrm{Ab}$ in our entire cohort of morbid obese patients was similar $(11 \%)$ to that found in the general population, as exemplified by figures reported by NHANES III (21). The female gender prevalence of positive test for Thyr$\mathrm{Ab}$ (14 and 3\% in women and men respectively) was also similar to that reported in the literature (21). These data indicate that patients with morbid obesity do develop thyroid autoantibodies just like normo-weight subjects.

Furthermore, as suggested by a recent review, the strongest epidemiological evidence for lowering the TSH normal range is the fact that euthyroid subjects with TSH between 3 and $4.5 \mathrm{mIU} / \mathrm{l}$ show higher rates of thyroid $\mathrm{Ab}$ positivity and of progression to overt hypothyroidism (19), in contrast to the finding of raised TSH levels without $A b$ positivity observed in morbid obese patients.

The fact that our obese patients with a raised TSH did not receive previous treatments with $\mathrm{LT}_{4}$ rules out the possibility that thyroid replacement therapy might have reduced the levels of circulating thyroid $A b(22)$.

The finding that the rate of positive tests for Thyr-Ab was low in our obese patients with a raised serum TSH, but not in the general population of subjects with morbid obesity, would support the view that thyroid autoimmunity is not responsible for the alteration of TSH and free thyroid hormones.

The observation that a female gender prevalence among morbid obese patients with hypothyroidism was found only in the subgroup with thyroid Ab positivity, but not in those with negative tests for thyroid $A b$, further supports the non-autoimmune etiology of thyroid function abnormalities in the latter subgroup of patients.

Comparison of serum thyroid function parameters between obese subjects with a raised TSH and normoweight patients matched for their serum level of TSH allowed further insights. Indeed, obese patients, at variance with normo-weight hypothyroid controls, had a similar $\mathrm{FT}_{4} / \mathrm{FT}_{3}$ ratio when compared with euthyroid normo-weights subjects. Taken together all of these data make the diagnosis of hypothyroidism strongly questionable, at least in morbid obese patients with negative tests for serum Tg-Ab and TPO-Ab.

The above considerations would allow hypothesizing that in patients with morbid obesity the raised serum levels of TSH may be independent of thyroid function, and that the interaction between TSH and adipose tissue occurs on a circuit different from that involved in thyroid regulation. Further indirect evidence supporting the above hypothesis derives from the reversibility of $\mathrm{SH}$ in morbid obese patients following weight loss induced by bariatric surgery (23) and from the observation that some peripheral indices of impaired thyroid function do not significantly differ between morbid obese patients with $\mathrm{SH}$ and those who are euthyroid $(24,25)$.

The observational data and the cross-sectional design of the current study together with the lack of routine thyroid ultrasound scans do not provide insights into the mechanisms responsible for the raised serum TSH observed in thyroid $\mathrm{Ab}$ negative patients with morbid obesity. However, there could be plausible biological explanations sustaining our findings and those of previous studies, which point to the same direction. In a phylogenetic hypothesis, individuals showing lower serum levels of $\mathrm{FT}_{4}$ and $\mathrm{FT}_{3}$ might belong to a population with a lower basal energy expenditure rate, which has been highly selected through evolution, and therefore would be more prone to develop morbid obesity. In their complex, our data and those of the literature would suggest that fat accumulation is associated with an increase in TSH levels, and that the control of TSH secretion by free thyroid hormones is impaired in morbid obesity, possibly as an adaptive thermogenic phenomenon.

In conclusion, the results of our study indicate that: increased serum levels of TSH and decreased concentrations of FT and FT are associated with morbid obesity; morbid obese patients have an increased prevalence of raised serum TSH, which in most cases is not accompanied by circulating thyroid $\mathrm{Ab}$; the finding of a thyroid hormone profile suggestive of SH may not indicate true hypothyroidism in patients with morbid obesity.

Future studies taking into account the peripheral indices of thyroid hypofunction will be required to further characterize the clinical significance of a raised serum TSH in morbid obese patients.

\section{Declaration of interest}

The authors declare that there is no conflict of interest that could be perceived as prejudicing the impartiality of the research reported. 


\section{Funding}

The experiments reported in this paper were supported in part by the funds from Progetto Ricerca Finalizzata 2005 'Rete Obesità' Italian Ministry of Health.

\section{References}

1 Bessesen DH. Update on obesity. Journal of Clinical Endocrinology and Metabolism 200893 2027-2034.

2 Ogden CL, Carroll MD, Curtin LR, McDowell MA, Tabak CJ \& Flegal KM. Prevalence of overweight and obesity in the United States, 1999-2004. Journal of the American Medical Association 2006295 1549-1555.

3 Haslam DW \& James WP. Obesity. Lancet 2005366 1197-1209.

4 Maes HH, Neale MC \& Eaves LJ. Genetic and environmental factors in relative body weight and human adiposity. Behavior Genetics 199727 325-351.

$5 \mathrm{Li} \mathrm{Z}$, Bowerman S \& Heber D. Health ramifications of the obesity epidemic. Surgical Clinics of North America $200585681-701$.

6 Ezzati M, Lopez AD, Rodgers A, Vander Hoorn S \& Murray CJ. Comparative Risk Assessment Collaborating Group. Selected major risk factors and global and regional burden of disease. Lancet 2002 360 1347-1360.

7 Olshansky SJ, Passaro DJ, Hershow RC, Layden J, Carnes BA, Brody J, Hayflick L, Butler RN, Allison DB \& Ludwig DS. A potential decline in life expectancy in the United States in the 21st century. New England Journal of Medicine 2005352 1138-1145.

8 DeMaria EJ. Bariatric surgery for morbid obesity. New England Journal of Medicine 2007356 2176-2183.

9 Kokkoris P \& Pi-Sunyer FX. Obesity and endocrine disease. Endocrinology and Metabolism Clinics of North America 200332 895-914.

10 Douyon L \& Schteingart D. Effect of obesity and starvation on thyroid hormone, growth hormone, and cortisol secretion. Endocrinology and Metabolism Clinics of North America 200231 173-189.

11 Sari R, Kemal Balci M, Altunbas H \& Karayalcin U. The effect of body weight and weight loss on thyroid volume and function in obese women. Clinical Endocrinology $200359258-262$.

12 Michalaki MA, Vagenakis AG, Leonardou AS, Argentou MN, Habeos IG, Makri MG, Psyrogiannis AI, Kalfarentzos FE \& Kyriazopoulou VE. Thyroid function in humans with morbid obesity. Thyroid $20061673-78$.

13 Manji N, Boelaert K, Sheppard MC, Holder RL, Gough SC \& Franklyn JA. Lack of association between serum TSH or free T4 and body mass index in euthyroid subjects. Clinical Endocrinology 200664 125-128.

14 De Pergola G, Ciampolillo A, Paolotti S, Trerotoli P \& Giorgino R. Free triiodothyronine and thyroid stimulating hormone are directly associated with waist circumference, independently of insulin resistance, metabolic parameters and blood pressure in overweight and obese women. Clinical Endocrinology 200767 265-269.

15 Bastemir M, Akin F, Alkis E \& Kaptanoglu B. Obesity is associated with increased serum TSH level, independent of thyroid function. Swiss Medical Weekly 2007137 431-434.

16 Knudsen N, Laurberg P, Rasmussen LB, Bulow I, Perrild H, Ovesen L \& Jorgensen T. Small differences in thyroid function may be important for body mass index and the occurrence of obesity in the population. Journal of Clinical Endocrinology and Metabolism 200590 4019-4024.

17 Schaffler A, Binart N, Scholmerich J \& Buchler C. Hypothesis paper brain talks with fat - evidence for a hypothalamic-pituitaryadipose axis? Neuropeptides 200539 363-367.

18 Pontikides N \& Krassas GE. Basic endocrine products of adipose tissue in states of thyroid dysfunction. Thyroid $2007 \mathbf{1 7} 421-431$.

19 Biondi B \& Cooper DS. The clinical significance of subclinical thyroid dysfunction. Endocrine Reviews 200829 76-131.

20 Ortega E, Koska J, Pannacciulli N, Bunt JC \& Krakoff J. Free triiodothyronine plasma concentrations are positively associated with insulin secretion in euthyroid individuals. European Journal of Endocrinology 2008158 217-221.

21 Spencer CA, Hollowell JG, Kazarosyan M \& Braverman LE. National Health and Nutrition Examination Survey III thyroidstimulating hormone (TSH)-thyroperoxidase antibody relationships demonstrate that TSH upper reference limits may be skewed by occult thyroid dysfunction. Journal of Clinical Endocrinology and Metabolism 200792 4236-4240.

22 O'Leary PC, Feddema PH, Michelangeli VP, Leedman PJ, Chew GT, Knuiman M, Kaye J \& Walsh JP. Investigations of thyroid hormones and antibodies based on a community health survey: the Busselton thyroid study. Clinical Endocrinology 2006 64 97-104.

23 Chikunguwo S, Brethauer S, Nirujogi V, Pitt T, Udomsawaengsup S, Chand B \& Schauer P. Influence of obesity and surgical weight loss on thyroid hormone levels. Surgery for Obesity and Related Diseases 20073 631-635.

24 Tagliaferri M. Berselli ME, Calò G, Minocci A, Savia G, Petroni ML Viberti GC \& Liuzzi A. Subclinical hypothyroidism in obese patients: relation to resting energy expenditure, serum leptin, body composition, and lipid profile. Obesity Research 20019196 201.

25 Reinehr T, de Sousa G \& Andler W. Hyperthyrotropinemia in obese children is reversible after weight loss and is not related to lipids. Journal of Clinical Endocrinology and Metabolism 2006913088 3091 .

Received 1 December 2008

Accepted 3 December 2008 